\title{
Methyl Group Rotation and Tunnelling in Crystals of 5-Membered Ring Molecules
}

\author{
A.-S. Montjoie, W. Müller-Warmuth, and Hildegard Stiller \\ Institut für Physikalische Chemie der Westfälischen Wilhelms-Universität, D-4400 Münster \\ J. Stanislawski \\ Institut für Festkörperforschung der KFA Jülich, D-5170 Jülich
}

Z. Naturforsch. 43 a, 35-42 (1988); received October 1, 1987

\begin{abstract}
${ }^{1} \mathrm{H}$ NMR spin-lattice relaxation times $T_{1}$ and - if accessible - level-crossing peaks and inelastic neutron scattering spectra have been measured for solid 2- and 3-methylfuran, 2- and 3-methylthiophene, 3- and 4-methylpyrazole, 1-methylimidazole, and 5-methylisoxazole. From the tunnel splittings, the torsional excitations and the NMR relaxation rates, the molecular dynamics of the methyl rotators has been evaluated between the limits of quantum tunnelling at low temperatures and thermally activated random reorientation at elevated temperatures.
\end{abstract}

\section{Introduction}

Molecular crystals formed of methyl substituted 5-membered heterocyclic rings represent an interesting example of rotator systems where the hindering potential is predominantly of intramolecular origin. The potential barriers which determine the reorientation of the $\mathrm{CH}_{3}$ groups are in general of the same order of magnitude in the free molecule [1] and in the solid. The barriers are furthermore of intermediate size, so that rotational tunnelling plays a role, and comprehensive information may be obtained from NMR $T_{1}$-measurements and level - crossing spectroscopy.

We have shown in a preceding paper [2] that the rotational potential and the details of the motional behaviour can be completely determined from proton NMR experiments if the barriers are in between about 3 and $7 \mathrm{~kJ} / \mathrm{mol}$. At low temperatures the methyl rotation is dominated by quantum mechanical tunnelling; the tunnel frequency is immediately measured by applying a field-cycling technique to search for a magnetic field in which the Zeeman and tunnel splittings match. Measurements of the torsional excitation from the ground state by inelastic neutron scattering (INS) yield additional information on the $\mathrm{CH}_{3}$ rotor. At elevated temperatures the rotation passes over to thermally activated classical reorientation; the activation energy and correlation time for this motion are obtained from the temperature dependence of $T_{1}^{-1}$ as usual. It is furthermore well known that for the whole temperature range both the spin-lattice relaxation rate and the correlation time can be described by a uniform model which contains both limits [3, 4].

In the present study the following methyl substituted materials have been examined:
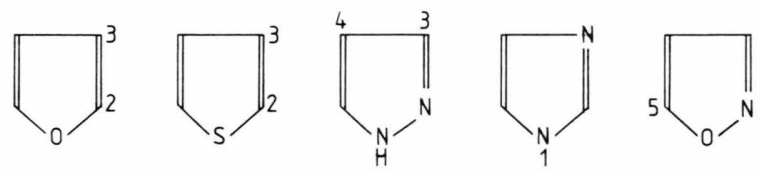

furan thiophene pyrazole imidazole isoxazole

The numerals denote the position of the $\mathrm{CH}_{3}$ group. We measured the temperature dependence of the relaxation rate $T_{1}^{-1}$ at 15 and $30 \mathrm{MHz}$ and registered its field dependence at very low temperatures by application of the field-cycling technique. The energy separation $E_{01}$ between the torsional ground and first excited state was observed by INS.

\section{Experimental}

The materials were purchased from Aldrich, Fluka, or Riedel-de Haen companies. 3-methylfuran was prepared in the Institute of Organic Chemistry of this University. The compounds were purified by distillation if necessary and carefully dried with molecular sieves. Before sealing the glass ampoules oxygen was removed from the samples by several freeze-pumpthaw cycles. 
The spin-lattice relaxation time $T_{1}$ was measured in the same way as outlined in the preceding paper [2]. $90^{\circ}-\tau-90^{\circ}$ pulse sequences were applied and $T_{1}$ was obtained from the initial slope of the plot $\ln \left\{\left(M_{0}-M_{z}\right) / M_{0}\right\}$ vs. time. The accuracy of $T_{1}^{-1}$ suffers from the non-exponentiality of this function and is only of the order of $\pm 10 \%$ or even less in unfavourable cases. The temperature was controlled by continuous flow liquid helium cryostats with PID regulators. It was independently measured near the sample by $\mathrm{Ni}-\mathrm{Cr} / \mathrm{Au}-0.02$ at $\% \mathrm{Fe}$ - thermocouples. The accuracy of the temperature measurement is $\pm 0.5 \mathrm{~K}$.

To apply the method of level-crossing spectroscopy we used the field-cycling technique $[5,6]$ and supplemented a pulsed NMR spectrometer by an appropriate home-made device. In the experiment the proton magnetization $M_{z}$ is first saturated by a train of radiofrequency pulses at a given magnetic field value $B_{0}$ (corresponding to a NMR frequency of 15 or $30 \mathrm{MHz}$ ). Then the magnetic field is switched to a new value $B_{r}$ where the spin system is offered the opportunity to relax. The field is afterwards returned to its original value $B_{0}$, and $M_{z}$ is measured by application of a $90^{\circ}$ pulse. This procedure is repeated many times for different $B_{r}$ values where the whole magnetic field range is passed in small steps. At magnetic field values $B_{r}$ such that a level-crossing condition $\left(\omega_{\mathrm{t}}^{0}=\omega_{0}, 2 \omega_{0}\right)$ is fulfilled, effective resonant exchange of energy between the proton spin and the tunnelling system occurs, which is indicated by an increase of $T_{1}^{-1}$ and a re-establishment of $M_{z}$. The procedure to search for level-crossing peaks is fully automated by computer control and a Hall stabilization unit for the field.

The method works only at very low sample temperatures where $T_{1}$ is sufficiently long in the absence of level-crossing. It is furthermore important to prepare the system after each cycle in a way that the tunnel reservoir is cold as compared with the "hot" spin reservoir; otherwise no exchange of energy would be observable. Before starting an experimental cycle, either a rather long time is needed for the recovery of the tunnel system, or this process must be accellerated by proper precooling procedures. We also realized that it is not possible to observe both level-crossing peaks within one passage, since after an energy exchange has happened the tunnel reservoir is saturated.

The resulting field-cycling scans (Fig. 1) are quite different as to the intensity and width of the resonances. The peaks of Figs. $1 \mathrm{a}$ and $1 \mathrm{~b}$ are assigned to
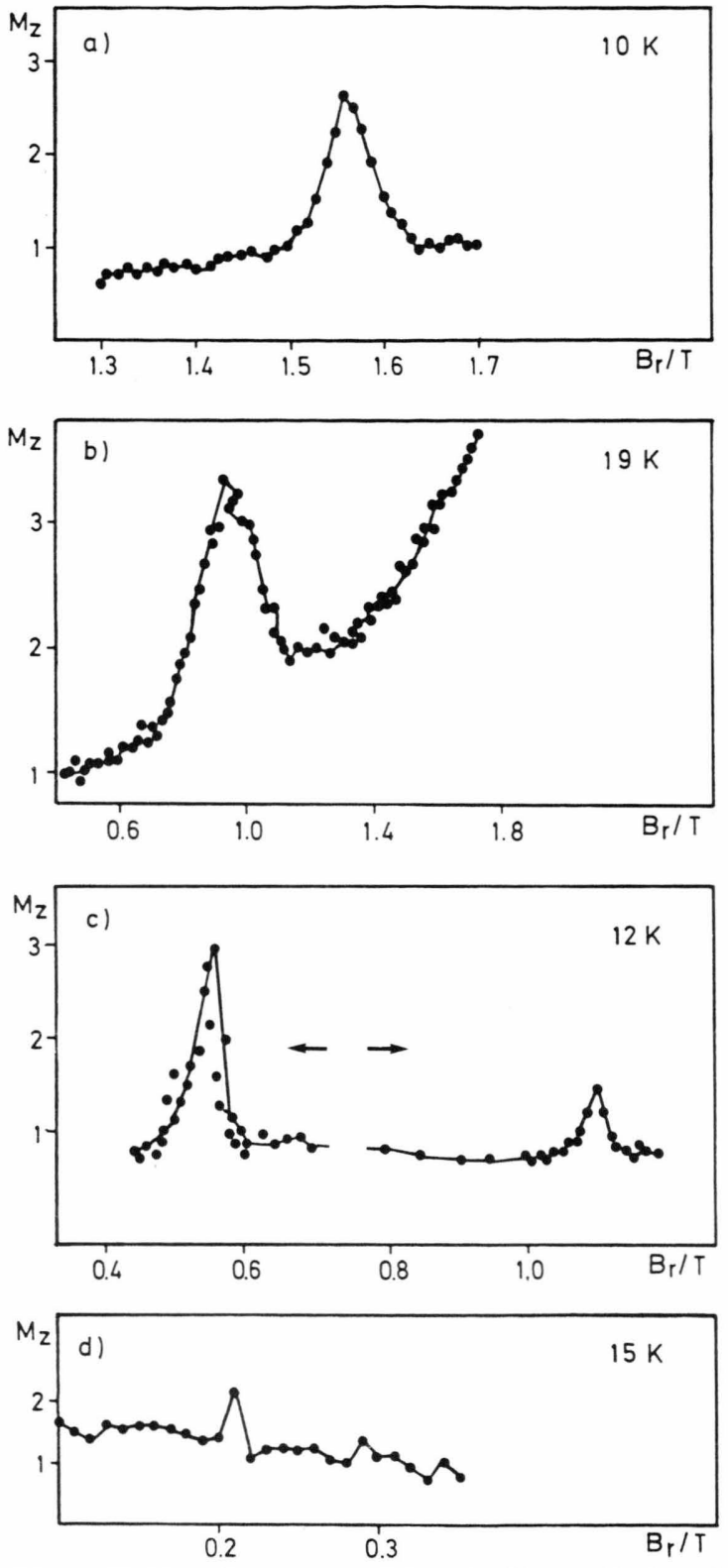

Fig. 1. Field-cycling scans of 3-methylfuran (a), 1-methylimidazole (b), 2-methylfuran (c), and 5-methylisoxazole (d).

the condition $\omega_{\mathrm{t}}^{0}=\omega_{0}$, that of Fig. $1 \mathrm{~d}$ to $\omega_{\mathrm{t}}^{0}=2 \omega_{0}$. In Fig. $1 \mathrm{c}$ both peaks are observed, but two separate scans were applied. The extremely weak peak of 5 -methylisoxazole was reproduced many times. The numerical values of $\omega_{\mathrm{t}}^{0}$ derived from the level-crossing experiments are listed in Table 1 of the next chapter.

INS was applied to determine the energy difference $E_{01}$ between the ground and first excited torsional 
state. The spectra were measured with the thermal time of flight spectrometer SV22 [7] at the FRJ2 reactor in Jülich using various combinations of incoming neutron energy and energy resolution. They were taken at different sample temperatures since the torsional excitations have to be distinguished from other phonon peaks by their broadening and weakening upon increasing the temperature. Figures 2 and 3 show examples of such measurements.

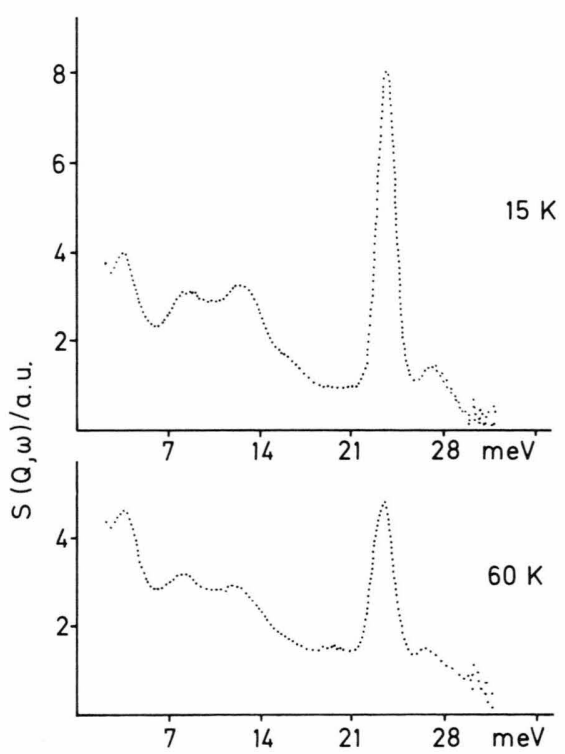

Fig. 2. Neutron scattering function of 5-methylisoxazole as derived from the time of flight spectra with a neutron wavelength of $1,5 \AA$ and an energy resolution of $2 \mathrm{meV}$. All detectors between scattering angles of $26^{\circ}$ and $126^{\circ}$ are summed up. The temperature dependence is used to discriminate torsional from phonon peaks.

\section{Results}

\section{1. $T_{1}$-measurements}

Figures 4 to 10 show the observed temperature dependences of ${ }^{1} \mathrm{H} \mathrm{T}_{1}^{-1}$ for the various crystals measured at two fixed NMR frequencies $\omega_{0}$. The solid lines correspond to curve fittings based on Haupt's equation [8]

$$
\begin{aligned}
\frac{1}{T_{1}}= & C_{\mathrm{AE}} \sum_{-2}^{+2} \frac{n^{2} \tau_{\mathrm{c}}}{1+\left(\omega_{\mathrm{t}}+n \omega_{0}\right)^{2} \tau_{\mathrm{c}}^{2}} \\
& +C_{\mathrm{EE}} \sum_{1}^{2} \frac{n^{2} \tau_{\mathrm{c}}}{1+n^{2} \omega_{0}^{2} \tau_{\mathrm{c}}^{2}} .
\end{aligned}
$$

The relaxation constants $C_{\mathrm{AE}}$ and $C_{\mathrm{EE}}$ account for dipole-dipole interactions which are connected with a change of symmetry of the $\mathrm{CH}_{3}$ rotor, either from $A$ to $E$ or from $E_{\mathrm{a}}$ to $E_{\mathrm{b}}$. As shown in more detail in the

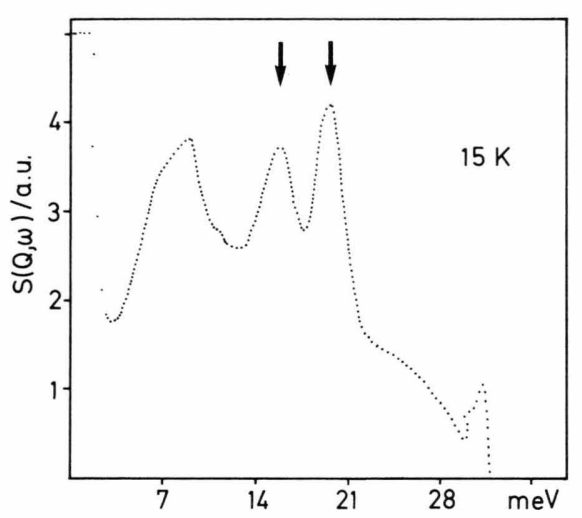

Fig. 3. Neutron scattering function of 1-methylimidazole. The conditions are the same as those of Fig. 2. Two torsional peaks are visible. They are broadened at higher temperature (not shown).

Table 1. Experimental results for the various materials either obtained directly from NMR and INS measurements or derived

\begin{tabular}{|c|c|c|c|c|c|c|c|}
\hline \multirow[t]{2}{*}{ No. } & \multirow[t]{2}{*}{ Molecular crystal } & \multicolumn{2}{|c|}{ Direct measurement } & \multicolumn{4}{|c|}{$T_{1}$-Fitting } \\
\hline & & $h \omega_{\mathrm{t}}^{0} / \mu \mathrm{eV}$ & $E_{01} / \mathrm{kJ} \mathrm{mol}^{-1}$ & $h \omega_{\mathrm{t}}(T) / \mu e V(K)$ & $E_{\mathrm{A}} / \mathrm{kJ} \mathrm{mol}^{-1}$ & $\tau_{0}^{\prime} / 10^{-13} \mathrm{~s}$ & $a / 10^{-11} \mathrm{~K}^{-6}$ \\
\hline 1 & 2-methylthiophene & & 0.48 & $17.6 \quad(53)$ & 2.7 & 0.76 & \\
\hline 2 & 3-methylpyrazole & & & $2.7 \quad(53)$ & 3.1 & 2.1 & \\
\hline 3 & 4-methylpyrazole & & 1.23 & $0.56 \quad(48)$ & 3.8 & 1.2 & 28 \\
\hline 4 & 3-methylthiophene & 0.53 & 1.72 & $0.25(51)$ & 4.5 & 5.3 & 6 \\
\hline \multirow[t]{2}{*}{5} & 1-methylimidazole (1) & & 1.51 & 0.25 (47) & 4.7 & 1.4 & 21 \\
\hline & 1-methylimidazole (2) & 0.33 & 1.88 & & & & \\
\hline 6 & 3-methylfuran & 0.55 & 1.95 & $0.25(45)$ & 5.0 & 7.7 & 15 \\
\hline 7 & 2-methylfuran & 0.19 & 1.79 & $0.062(45)$ & 5.4 & 2.0 & 3 \\
\hline 8 & 5-methylisoxazole & 0.039 & 2.27 & & 7.4 & 1.8 & \\
\hline
\end{tabular}
from the NMR $T_{1}$-measurements. 
preceding paper [2], in the fits the temperature dependences of the tunnel frequency $\omega_{\mathrm{t}}$ and the correlation time $\tau_{\mathrm{c}}$ are approximated by the relations

$$
\begin{aligned}
\omega_{\mathrm{t}}(T)= & \frac{\omega_{\mathrm{t}}^{0}}{1+a T^{6}}, \\
\tau_{\mathrm{c}}^{-1}(T)= & \left(\tau_{0}^{\prime}\right)^{-1} \exp \left\{-E_{\mathrm{A}}^{\prime} / R T\right\} \\
& +\left(\tau_{0}^{\prime \prime}\right)^{-1} \exp \left\{-E_{\mathrm{A}}^{\prime \prime} / R T\right\} .
\end{aligned}
$$

The parameters $a, \tau_{0}^{\prime}, E_{\mathrm{A}}^{\prime}, E_{\mathrm{A}}^{\prime \prime}$ are characteristic of the respective material. $E_{\mathrm{A}}^{\prime}$ may be identified with the activation energy $E_{\mathrm{A}}$ for the classical reorientation with the rate $\tau_{\mathrm{c}}^{-1}$. If the potential barrier is low, $E_{\mathrm{A}}^{\prime \prime}$ approaches $E_{01}$, the energy difference between the torsional ground and first excited state.

Equation (1) holds only in cases where all $\mathrm{CH}_{3}$ groups are equivalent. A weighted average of several equations like (1) has to be formed if there is more than one distinct rotator [9]. In this study no attempt was made to apply such a procedure, not even for 1-methylimidazole and 2-methylfuran where there is some evidence of non-equivalent methyl groups.

The numerical parameters obtained from the fitting procedure are listed in Table 1 together with the $\omega_{\mathrm{t}}^{0}$ and $E_{01}$ data from the field cycling and INS measurements.

\subsection{Tunnel splittings}

Large ground state splittings $\hbar \omega_{\mathrm{t}}^{0}$ cannot be detected directly by NMR. The pronounced frequency independent relaxation maxima of the first three materials (Figs. 4, 5, 6), however, give a lower limit $\hbar \omega_{\mathrm{t}}(T)$ of the tunnel frequency (see Table 1$) . \hbar \omega_{\mathrm{t}}^{0}$ may then be estimated from (2). An attempt to observe $\hbar \omega_{t}^{0}$ for 2-methylthiophene directly by INS was not successful [10].

For the remaining five materials the ground state tunnel splittings were determined by field-cycling spectroscopy and listed in Table 1. Tunnelling frequencies at somewhat elevated temperatures, $\omega_{\mathrm{t}}(T)$, appear in most of the $\mathrm{T}_{1}^{-1}$ curves (Figs. 7, 8, 9) as an extra peak by level crossing, if $\omega_{t}=\omega_{0}$ or $2 \omega_{0}$ holds.

\subsection{Torsional excitations}

With the exception of 3-methylpyrazole we succeeded to obtain $E_{01}$ from the INS spectra of all the materials. In 1-methylimidazole (Fig. 3) two torsional energies were identified, in 2-methylfuran even four;

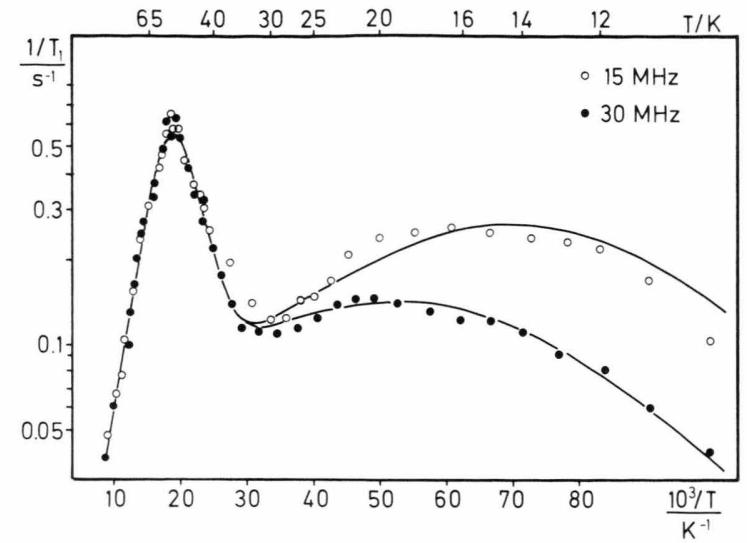

Fig. 4. Proton spin-lattice relaxation rates plotted against reciprocal temperatures for 2-methylthiophene. The solid lines correspond to the fit explained in the text. The parameters derived from this fit are given in Table 1.

this is an indication of the existence of non-equivalent methyl groups. In all other cases only one torsional transition was observed. For 3-methylfuran, however, the intensity was very weak. The numerical values are listed in Table 1.

\section{Discussion}

\subsection{Hindering potential}

The single particle rotational potential which hinders the motion, can be derived from the data of Table 1. We truncate the Fourier expansion with the basic threefold symmetry of the $\mathrm{CH}_{3}$ group to the first two terms, as usual,

$$
\begin{aligned}
V(\varphi)= & \frac{V_{3}}{2}\left[1+(-1)^{k} \cos 3 \varphi\right] \\
& +\frac{V_{6}}{2}\left[1+(-1)^{k} \cos 6 \varphi\right],
\end{aligned}
$$

and take advantage of the tabulated eigenvalues of the Schrödinger equation for this potential [11]. If at least two of the three values $E_{\mathrm{A}}, E_{01}, \hbar \omega_{\mathrm{t}}^{0}$ are available, $V_{3}$ and $V_{6}$ can be determined. Table 2 presents the result in terms of $V_{\mathrm{s}}=V_{3}+V_{6}$ and $\delta=V_{3} / V_{\mathrm{s}}$. Very often two solutions exist; for systematical reasons we then always favour the solution with a dominant threefold potential $[2,4]$.

\subsection{2-methylthiophene and 3-methylpyrazole}

Both temperature dependences of $T_{1}^{-1}$ (Figs. 4, 5) are characteristic of the condition $\omega_{t} \gg \omega_{0}$ where all 


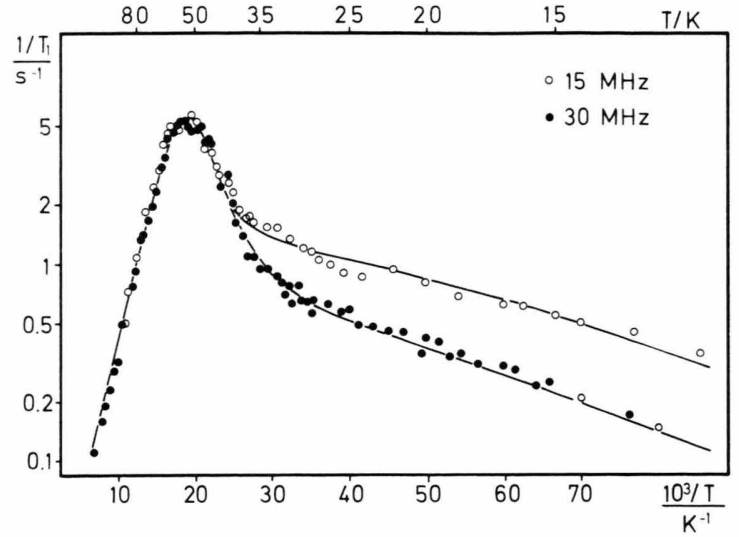

Fig. 5. Same as Fig. 4, but for 3-methylpyrazole.

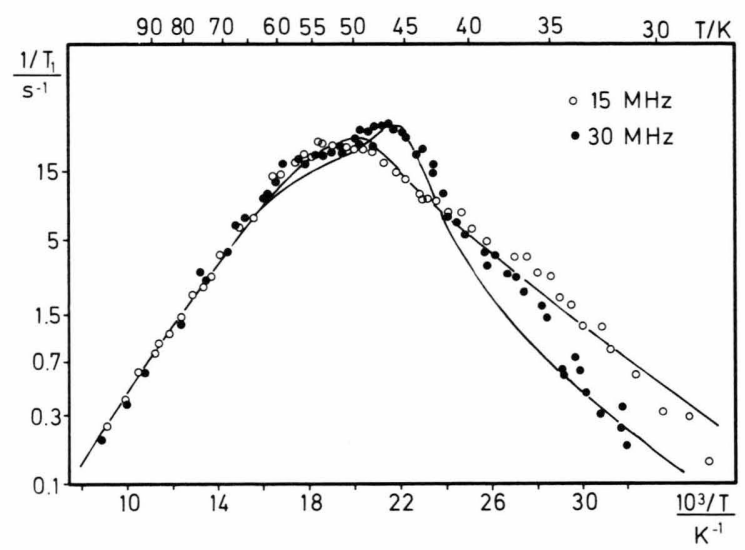

Fig. 7. Same as Fig. 4, but for 1-methylimidazole.

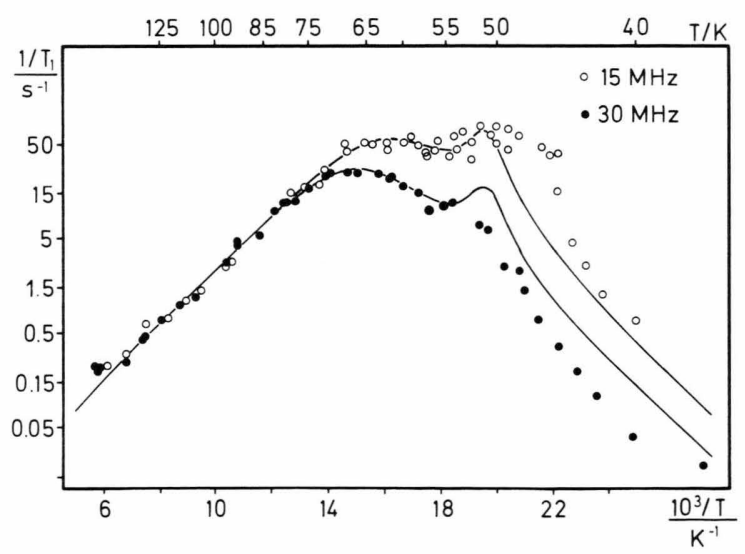

Fig. 9. Same as Fig. 4, but for 2-methylfuran.

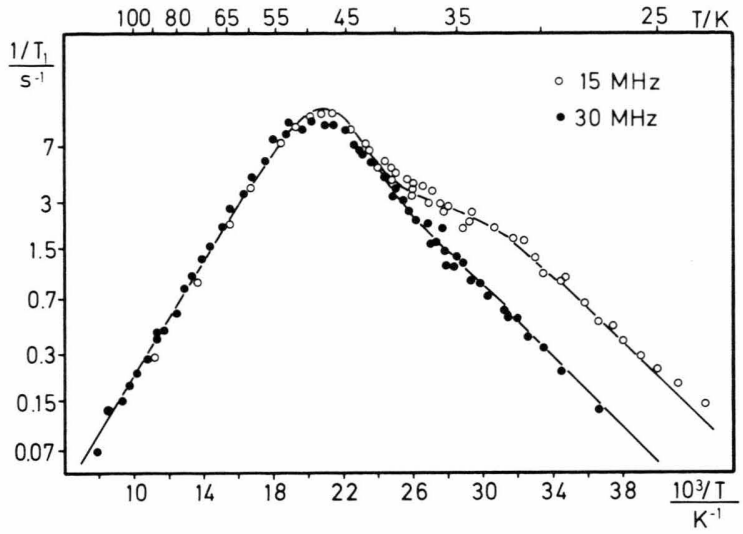

Fig. 6. Same as Fig. 4, but for 4-methylpyrazole.

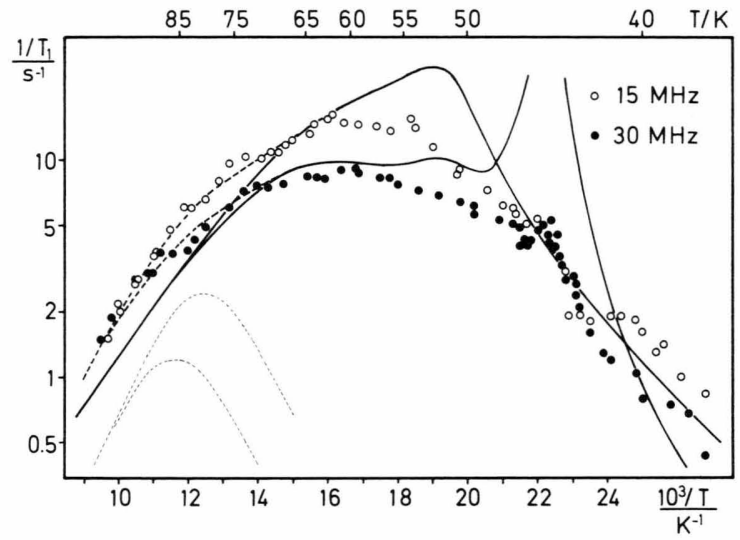

Fig. 8. Same as Fig. 4, but for 3-methylfuran.

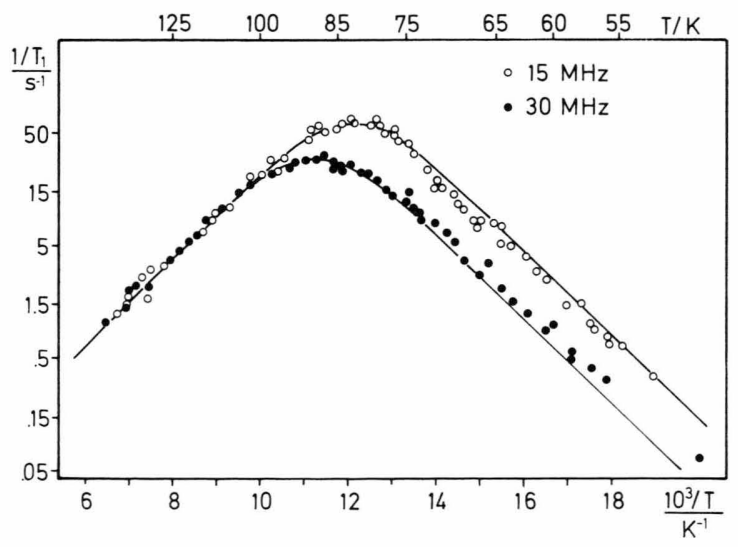

Fig. 10. Same as Fig. 4, but for 5-methylisoxazole. 
the terms of the first sum of (1) coincide in one curve with a large maximum. The second sum of (1) is less important $\left(C_{\mathrm{AE}} \gg C_{\mathrm{EE}}\right)$, showing that intramethyl interactions dominate. Comparison of the activation energies $E_{\mathrm{A}}$ with the barriers which were measured by microwave spectroscopy in the gaseous state ( 2.32 and $1.8 \mathrm{~kJ} / \mathrm{mol}$ ) [1, 12] points out that the hindrance of the rotation is mostly of intramolecular origin.

The apparent low temperature activation energy $E_{\mathrm{A}}^{\prime \prime}$ of the $T_{1}^{-1}$ vs $T^{-1}$ curves may be considered as a measure of the first torsional excitation $E_{01}$, since the barriers are low [2]. Taking $E_{\mathrm{A}}$ and $E_{01}$ as known, $h \omega_{\mathrm{t}}^{0}$ can be calculated from the Schrödinger equation with a potential (4); it becomes 46 and $23 \mu \mathrm{eV}$, respectively. There is satisfactory agreement with the reduced values of the tunnel splittings $h \omega_{\mathrm{t}}(53 \mathrm{~K})$ listed in Table 1. In contrast to most other materials that were examined in the past there occurs an important sixfold contribution to the potential in both cases, see Table 2. This could be a consequence of the nature of the largely intramolecular hindrance and the symmetry of the molecules. Since the tunnel splitting was not measured directly, however, the accuracy of this evaluation is less good than in the other cases.

\subsection{4-methylpyrazole and 3-methylthiophene}

The activation energies and tunnel splittings lie already in a range where those anomalies of the $T_{1}^{-1}$ behaviour appear (Fig. 6 and Fig. 7 of [2]) which are discussed at some length in the previous paper [2]. Quite good INS spectra indicate the existence of only one type of methyl group and yield reliable values of $E_{01}$. The apparent activation energies $E_{\mathrm{A}}^{\prime \prime}$ are no longer equal to $E_{01}$ as to be expected for barriers with $E_{\mathrm{A}} \gtrsim 3.5 \mathrm{~kJ} / \mathrm{mol}$. The potential (Table 2) is assumed to be predominantly threefold, in one case with an additional small broadening of the minima $(k=0)$, in the other one of the maxima $(k=1)$. The data for 3-methylthiophene are particularly well confirmed since all three parameters $\left(E_{\mathrm{A}}, h \omega_{\mathrm{t}}^{0}, E_{01}\right)$ have been directly measured and the potential is overdetermined, i.e. the values are verified twice. For 4-methylpyrazole $h \omega_{\mathrm{t}}^{0}=2.6 \mu \mathrm{eV}$ is indirectly obtained from both the Schrödinger equation and the $T_{1}$-fit.

Microwave data are available for 3-methylthiophene [1, 13]; the intramolecular barrier of $3.09 \mathrm{~kJ} /$ mol measured in the free molecule has the same order of magnitude as the hindrance in the crystal. For the magnetic dipolar interaction intramethyl effects dominate as well.

\subsection{1-methylimidazole}

Two torsional peaks in the INS spectrum (Fig. 3) indicate the existence of non-equivalent methyl groups. The field cycling spectrum (Fig. $1 \mathrm{~b}$ ), on the other hand, suggests that there is also a second levelcrossing peak, however, at a field value which is not accessible. We assign the smaller $E_{01}$ to this latter resonance and to the first type of $\mathrm{CH}_{3}$ (1) groups. The pertinent tunnel splitting amounts to $0.85 \mu \mathrm{eV}$, in due agreement with $h \omega_{\mathrm{t}}(47 \mathrm{~K})=0.25 \mu \mathrm{eV}$. The larger value of $E_{01}$ corresponds well with the measured tunnel peak at $0.33 \mu \mathrm{eV}$, cf. Table 1 .

In Fig. 7 we have only shown a curve fit for the first type of methyl groups, and we assign the deviations at low temperatures to effects inherent in the other kind of $\mathrm{CH}_{3}$. Particularly, since the activation energies look quite similar, a clear discrimination of nonequivalent methyl groups is not possible from the $T_{1}$ data.

Both rotational potentials can be derived (Table 2); they are predominantly threefold, but the sixfold correction has a different phase factor.

\subsection{3-methylfuran}

The activation energy is in agreement with the intramolecular barrier of $4.55 \mathrm{~kJ} / \mathrm{mol}$ measured in the free molecule [1, 14]. The $T_{1}^{-1}$ dependence (Fig. 8) looks quite anomalous and can only be fitted approximatively. Between about 50 and $70 \mathrm{~K}$, and at $30 \mathrm{MHz}$ near $45 \mathrm{~K}$, the relaxation was extremely non-exponential implying a relatively large error and indicating the occurence of level-crossing maxima. One maximum is clearly distinguishable at $45 \mathrm{~K}$, other maxima broaden both curves in the region where also other relative maxima occur. The level-crossing peaks do not appear, however, in such a distinct way as predicted by the theory. The same was observed in previous studies [2]. The fit suffers furthermore from an additional mechanism of motion which produces frequency-dependent maxima at high temperatures and whose origin is not clear. We interpreted this in terms of an additional BPP curve with an activation energy of $6.7 \mathrm{~kJ} / \mathrm{mol}$, cf. Figure 8 .

The potential of Table 2 is not much different from that of the second type of methyl groups in 1-methylimidazole. The existence of non-equivalent methyl groups is not completely excluded since the INS spectrum is poor. 
Table 2. Rotational potentials for the various materials as derived from the experimental data.

\begin{tabular}{llllllll}
\hline Molecular crystal & \multicolumn{3}{c}{ Solution I } & & \multicolumn{3}{c}{ Solution II } \\
\cline { 2 - 3 } & $k$ & $V_{\mathrm{s}} / \mathrm{kJ} \mathrm{mol}^{-1}$ & $\delta$ & & $k$ & $V_{\mathrm{s}} / \mathrm{kJ} \mathrm{mol}^{-1}$ & $\delta$ \\
\hline 2-methylthiophene & 0 & 4.3 & 0.59 & & 1 & 4.2 & 0.13 \\
3-methylpyrazole & 0 & 5.0 & 0.50 & & 4.6 & 0.08 \\
4-methylpyrazole & 0 & 4.9 & 0.90 & & 6.0 & 0.23 \\
3-methylthiophene & 1 & 5.6 & 0.94 & 1 & 6.9 & 0.28 \\
1-methylimidazole (1) & 0 & 5.6 & 0.95 & 1 & 7.1 & 0.24 \\
1-methylimidazole (2) & 1 & 6.1 & 0.90 & 1 & 7.4 & 0.29 \\
3-methylfuran & 1 & 6 & 0.9 & 1 & 7.4 & 0.25 \\
2-methylfuran & 0,1 & 6.5 & 1 & 1 & 8.4 & 0.23 \\
5-methylisoxazole & 1 & 8.7 & 0.93 & 1 & 10.6 & 0.23 \\
\hline
\end{tabular}

\subsection{2-methylfuran}

Four torsional excitations have been observed, namely in addition to the value listed in Table 1 at energy transfers of $0.72,1.47$ and $2.03 \mathrm{~kJ} / \mathrm{mol}$. Only $E_{01}=1.79 \mathrm{~kJ} / \mathrm{mol}$ fits well into the data of $E_{\mathrm{A}}$ and $h \omega_{\mathrm{t}}^{0}$ and gives the purely threefold potential of Table 2. Again, the activation energy measured in the crystal is not far from $4.98 \mathrm{~kJ} / \mathrm{mol}$ for the molecule from microwave spectroscopy $[1,15]$.

$T_{1}^{-1}$ in Fig. 9 is distinguished by large nonexponential regions with the corresponding larger error limits. As in Fig. 7, the fit does not account for the non-equivalence of the methyl groups but uses only the values for that $\mathrm{CH}_{3}$ group which is considered in Tables 1 and 2.

\subsection{5-methylisoxazole}

The rotational potential (Table 2) is well established by the experimental values of $E_{\mathrm{A}}, \hbar \omega_{\mathrm{t}}^{0}$, and $E_{01}$. Among the materials examined 5-methylisoxazole has the largest barrier, different from what is known for the free molecule $(3.3 \mathrm{~kJ} / \mathrm{mol})$ [16].

Since the tunnelling frequency is no longer larger than the NMR frequency, the $T_{1}^{-1}$ vs. $T^{-1}$ curve of Fig. 10 is reminiscent of a classical BPP behaviour [17]. The information is correspondingly inferior.

\section{Conclusions}

Profitting from the procedure of NMR $T_{1}$-analyses outlined in the preceding paper [2], methyl tunnelling and reorientation in eight different crystals formed of 5 -membered ring molecules could be described. Levelcrossing experiments by field-cycling spectroscopy and inelastic neutron scattering resulted in values for the tunnel splitting and the first torsional excitation. The potential barriers are all of intermediate size and intramolecular hindrance dominates. The symmetry of the potential is in most cases not far from threefold, in two compounds there is an important sixfold contribution which gives rise to rather large tunnel splittings.

The observed spin-lattice relaxation rates are striking examples of the change in the temperature and frequency dependence with increasing potential barriers and decreasing tunnel splittings. The character of the curves passes from the limit of very large tunnelling frequencies (as compared with the NMR frequency) to the classical limit where tunnelling is no longer observable. Among the numerical parameters for the curve fittings, $\tau_{0}^{\prime} \approx 10^{-13} \mathrm{~s}$ and $E_{\mathrm{A}}$ describe the classical reorientation. $h \omega_{\mathrm{t}}^{0}$ and $E_{01}$ are important for the low temperature behaviour and for the determination of the potential. $\tau_{0}^{\prime \prime}$ and $E_{\mathrm{A}}^{\prime \prime}$ are only approximative parameters, which become well defined in the limit of low barriers. $h \omega_{\mathrm{t}}(T)$ and "a" inform on the temperature dependence of the tunnel splitting. The relaxation constants $C_{\mathrm{AE}}$ and $C_{\mathrm{EE}}$ are only a reliable measure of the importance of intra- and intermethyl interactions for the first five materials of Table 1. At lower barriers the two terms of (1) become rather similar, and finally a total relaxation constant $2 C_{\mathrm{AE}}+C_{\mathrm{EE}}$ results.

Figure 11 shows that the correlation between the experimental values of the tunnel splitting and the activation energy is remarkably good. The data fit well into the systematics already known for a large number of materials [4]. An analysis by Clough et al. [18], which was also given a theoretical basis [19], has revealed another interesting correlation, namely that between the tunnel splitting and the temperature $\theta_{\max }$ at which $T_{1}^{-1}$ has its (frequency dependent) maxi- 


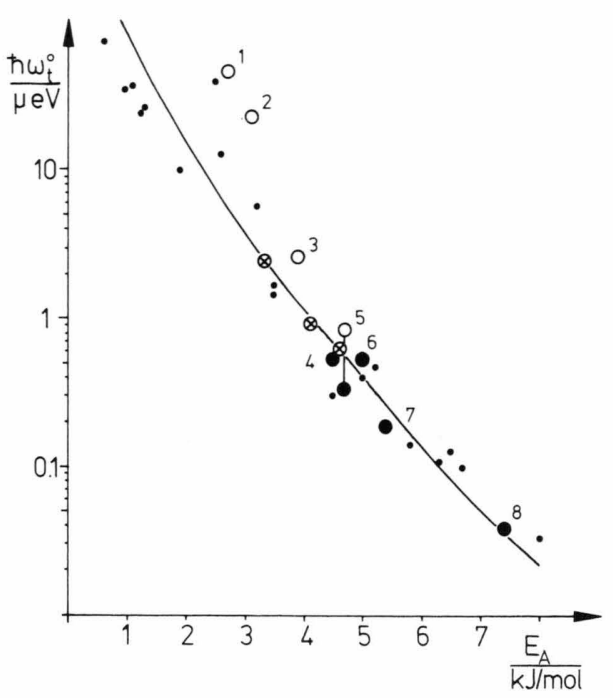

Fig. 11. The correlation of methyl tunnel splittings and activation energies as determined for various materials, where $\hbar \omega_{\mathrm{t}}^{0}$ was either directly measured (•) or derived from other parameters $(\bigcirc)$. $\otimes$ refer to the data of the previous paper [2], - characterizes previous measurements of this and other laboratories, cf. [4]. The line corresponds to the relationship expected for a purely threefold potential.

mum. Figure 12 demonstrates the existence of such a correlation for the materials of this study together with other data (not specified). In most cases the centres of the $T_{1}^{-1}$ maxima could not be judged by eye from the figures; the values of Fig. 12 were obtained from the computer fits. It may be noticed that materials with a preferentially sixfold potential fulfill this relationship as well. Moreover, if there is more than one chemically distinct $\mathrm{CH}_{3}$ group, the observed $\theta_{\max }$ refers only to one of the non-equivalent methyl groups (cf. compound no. 5) as to be expected. Like the relax-

[1] J. Sheridan, Microwave Spectra of Heterocyclic Molecules, in "Physical Methods in Heterocyclic Molecules", (A. R. Katritzky, ed.), Vol. 6, Academic Press, New York 1974.

[2] H. Langen, A.-S. Montjoie, W. Müller-Warmuth, and H. Stiller, Z. Naturforsch.42a, 1266 (1987).

[3] S. Clough, P. J. McDonald, and F. O. Zelaya, J. Phys. C.: Solid State Phys. 17, 4413 (1984).

[4] A.-S. Montjoie and W. Müller-Warmuth, Z. Naturforsch. $\mathbf{4 0 a}, 596$ (1985).

[5] P. van Hecke and J. Janssens, Phys. Rev. B 17, 2124 (1978).

[6] B. Gabrys, Mol. Phys. 51, 601 (1984), and references therein.

[7] H. Pohl and M. Prager, Internal Report JÜL-SPEZ-176 (1982).

[8] J. Haupt, Z. Naturforsch. 26a, 1578 (1971).

[9] M. Prager, K.-H. Dupree, and W. Müller-Warmuth, Z. Phys. B. - Condensed Matter 51, 309 (1983).

[10] M. Prager, private communication.

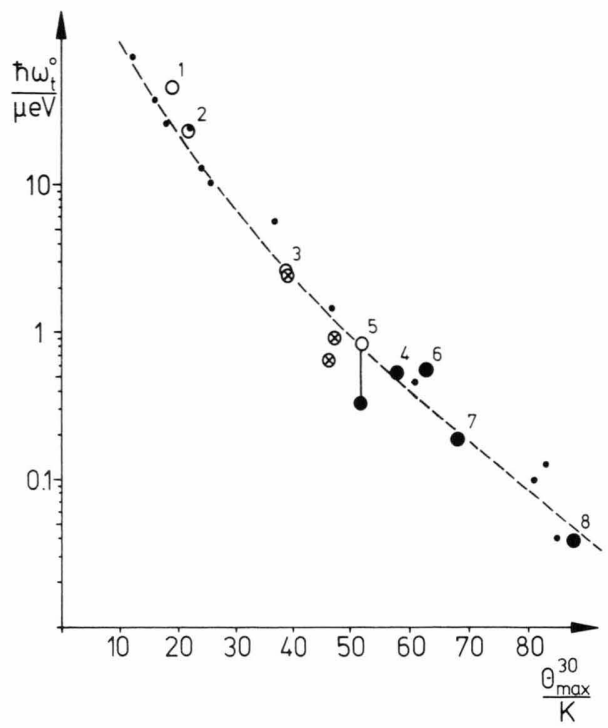

Fig. 12. The correlation of methyl tunnel splittings and the temperature $\theta_{\max }^{30}$ at which the spin-lattice relaxation rate has its frequency-dependent maximum when measured with an NMR frequency of $30 \mathrm{MHz}$. The symbols are the same as in Fig. 11; the line serves as a guide to the eye.

ation rate curves, the relationship of Fig. 12 demonstrates the correlation between quantum tunnelling and thermally activated motion.

\section{Acknowledgements}

We are indebted to Dipl.-Chem. H. Langen for his support to fit the curves and to Dr. M. Prager for his advice during the neutron scattering experiments. The financial support of this work by the Deutsche Forschungsgemeinschaft is gratefully acknowledged.

[11] R. F. Gloden, Euratom Reports EUR 4349 and EUR 4358 (1970) and supplements by M. Prager, Jülich.

[12] N. M. Pozdeev, L. N. Gunderova, and A. A. Shapkin, Opt. Spektrosk. 28, 254 (1970).

[13] T. Ogata and K. Kozima, J. Mol. Spectrosc. 42, 38 (1972).

[14] T. Ogata and K. Kozima, Bull. Chem. Soc. Japan 44, 2344 (1971).

[15] U. Andresen and H. Dreizler, Z. Naturforsch. 27 a, 705 (1972).

[16] S. L. Srivastava, Indian J. Phys. 51 B, 1 (1977).

[17] N. Bloembergen, E. M. Purcell, and R. V. Pound, Phys. Rev. 73, 679 (1948).

[18] S. Clough, A. Heidemann, A. J. Horsewill, J. D. Lewis, and M. N. J. Paley, J. Phys. C.: Solid State 14, L 525 (1981).

[19] S. Clough, A. Heidemann, A. J. Horsewill, J. D. Lewis, and M. N. J. Paley, J. Phys. C.: Solid State 15, 2495 (1982). 